\title{
ELEMENTARY PROOFS OF SOME ASYMPTOTIC RADIAL UNIQUENESS THEOREMS
}

\author{
ROBERT D. BERMAN
}

ABSTRACT. Elementary proofs of several generalizations of Tse's extension of an asymptotic radial uniqueness theorem of Barth and Schneider are given.

Let $\Delta=\{|z|<1\}$ and $C=\{|z|=1\}$. The following is an extension to meromorphic functions by Tse [5] of a theorem of Barth and Schneider [1].

THEOREM 1. Let $\mu$ be a positive monotone decreasing function with domain $[0,1)$ such that $\lim _{r \rightarrow 1} \mu(r)=0$. Let $S$ be a second category subset of $C$. If $f$ is a meromorphic function on $\Delta$ with the property that $f(r \eta)=o[\mu(r)]$ for each $\eta \in S$, then $f \equiv 0$.

Barth and Schneider's proof (for bounded analytic functions) depends on deep theorems of Mergelyan, Lusin-Privalov, and Collingwood. Tse's proof of Theorem 1 is based on the method of Barth and Schneider. An equivalent formulation [4, Corollary 2] of Theorem 1 may be obtained in a more straightforward manner as a corollary of a theorem of Rippon [4, Theorem 1]; however, this proof still relies on the Collingwood maximality theorem as well as results and methods needed for a proof of the Lusin-Privalov theorem. The proofs that we shall give to several generalizations of Theorem 1 are elementary and are based on the following.

CATEgORY PRINCIPLE. Let $S$ be a second category set. If $S=\cup_{n=1}^{\infty} F_{n}$ with each $F_{n}$ closed, then some $F_{n}$ contains a nonempty open set.

Proof. Since $E$ is of second category, some $F_{n}$ must be dense in an open set $U$. Since $F_{n}$ is closed, we have $U \subseteq F_{n}$ as required.

We turn now to our first generalization of Theorem 1. Let \&s be a continuum contained in $\bar{\Delta}=\{|z| \leqslant 1\}$ such that $\mathbb{S} \cap C=\{1\}$ and let $\mathscr{S}_{\eta}=\{\eta z: z \in \mathbb{S}\}$ for each $\eta \in C$. For $\mu$ a positive function with domain $[0,1)$ such that $\lim _{r \rightarrow 1} \mu(r)=0$ and $\eta \in C$, we shall write $f(z)=O[\mu(|z|)], z \in \mathscr{S}_{\eta}$, when $\lim \sup _{|z| \rightarrow 1}|\mathrm{f}(z)| / \mu(|z|)$ $<+\infty, z \in \mathbb{S}_{\eta} \cap \Delta$.

THEOREM 2. Let $\mu$ be a positive function with domain $[0,1)$ such that $\lim _{r \rightarrow 1} \mu(r)=0$ and $S$ a second category subset of $C$. If $f$ is a meromorphic function on $\Delta$ with the property that $f(z)=O[\mu(|z|)], z \in \mathbb{S}_{\eta}$, for each $\eta \in S$, then $f \equiv 0$.

Received by the editors March 31, 1982.

1980 Mathematics Subject Classification. Primary 30D40.

Key words and phrases. Asymptotic radial uniqueness, Barth-Schneider. 
Theorem 1 follows from Theorem 2 when $\mathbb{S}=[0,1]$.

Proof. Let $Z_{\mu}(f)=\left\{\eta \in C: f(z)=O[\mu(|z|)], z \in \mathbb{S}_{\eta}\right\}$. Then $Z_{\mu}(f)=\cup_{n=1}^{\infty} F_{n}$ where $F_{n}=\left\{\eta \in C:|f(z)| \leqslant n \mu(|z|), z \in \mathscr{S}_{\eta}\right.$ and $\left.1-\frac{1}{n} \leqslant|z|<1\right\}$ for each $n$. Now each $F_{n}$ is closed by the continuity of $f$. Since $Z_{\mu}(f)$ is of second category by assumption, there exists some $n$ for which $F_{n}$ contains a nonempty open arc $A$ (category principle). Note that the set $\cup_{\eta \in A} \mathscr{S}_{\eta} \cap\left\{1-\frac{1}{n} \leqslant|z|<1\right\}$ is a neighborhood (in $\Delta$ ) of each point of $A$. It follows from the definition of $F_{n}$ that $f$ is continuously 0 at each point of the $\operatorname{arc} A$. We conclude from the Schwarz reflection principle and the identity theorem that $f \equiv 0$. Theorem 2 is established.

Our next generalization extends Theorem 1 to the unit ball in $\mathbf{C}^{n}, n \geqslant 1$. Let $\Delta_{n}=\left\{z \in \mathbf{C}^{n}:\|z\|<1\right\}$ and $C_{n}=\left\{z \in \mathbf{C}^{n}:\|z\|=1\right\}$.

THEOREM 3. Let $\mu$ be as in Theorem 2 and $S$ a second category subset of $C_{n}$. If $f$ is meromorphic on $\Delta_{n}$ with $f(r \eta)=O[\mu(r)]$ for each $\eta \in S$, then $f \equiv 0$.

Proof. Except for the modification that $A$ is now an open subset of $C_{n}$ instead of an open $\operatorname{arc}$ of $C$, the proof proceeds as above (with $\&=[0,1]$ ) up to the conclusion that $f$ is continuously 0 at each point of $A$.

We show that $f \equiv 0$ as follows. Let $w \in \Delta_{n}$ and $\eta \in A$. There exists a univalent analytic map $\varphi$ defined on a neighborhood of $\bar{\Delta}_{n}$ mapping $\Delta_{n}$ onto itself such that $\varphi(w)=(0, \ldots, 0)$ and $\varphi(\eta)=(1,0, \ldots, 0)$. (Such a map $\varphi$ can be constructed explicitly using maps of the form

$$
\psi_{\beta}:\left(z_{1}, \ldots, z_{n}\right) \rightarrow\left(\frac{z_{1}-\beta}{1-\bar{\beta} z_{1}}, \frac{\sqrt{1-|\beta|^{2}}}{1-\bar{\beta} z_{1}} z_{2}, \ldots, \frac{\sqrt{1-|\beta|^{2}}}{1-\bar{\beta} z_{1}} z_{n}\right),
$$

$\left(z_{1}, \ldots, z_{n}\right) \in \Delta_{n}$ for $\beta \in \Delta$ and unitary linear transformations; cf. [2, p. 420].) Then $g(z)=f \circ \varphi^{-1}(z, 0, \ldots, 0)$ is a meromorphic function on $\Delta$ which is continuously 0 at each point of an open arc $I$ containing 1 and contained in $\{\xi \in C:(\xi, 0, \ldots, 0) \in$ $\varphi(A)\}$. It follows from the Schwarz reflection principle and the identity theorem that $g \equiv 0$. In particular, $f(w)=f \circ \varphi^{-1}(0, \ldots, 0)=g(0)=0$. Since $w \in \Delta_{n}$ was arbitrary, we conclude that $f \equiv 0$. This completes the proof of Theorem 3 .

It is possible to generalize Theorem 3 in a way analogous to that in which Theorem 2 generalizes Theorem 1, though care must be taken in framing a workable definition of rotating a continuum when $n>1$. One possibility is to phrase such a definition in terms of group actions on the sphere $C_{n}$. An analogue of such a generalization for a half space $H_{n}=\left\{\left(z_{1}, \ldots, z_{n}\right) \in \mathbf{C}^{n}: \operatorname{Im} z_{n}>0\right\}$ is more easily formulated and proved; however, we shall not pursue these generalizations here.

Rippon [3, Theorem 3] has given a subharmonic analogue of Theorem 1 for the half space $D=\left\{\left(x_{1}, \ldots, x_{n}\right) \in \mathbf{R}^{n}: x_{n}>0\right\}$ when $n>2$. His proof depends on a generalized form of the Collingwood maximality theorem for "fine continuous" functions (a class containing the subharmonic functions) proved in the same paper [3, Theorem 1]. It is also noted that his arguments apply equally well to the half plane. The following analogue of Rippon's result for continuous subharmonic functions in the disk $\Delta$ may be proved along the same lines as our proof of Theorem 2. We assume that $\mathscr{S S}^{\text {and }} \mathfrak{S S}_{\eta}, \eta \in C$, are as preceding that theorem. 
THEOREM 4. Let $\nu$ be a real-valued function with domain $[0,1)$ such that $\lim _{r \rightarrow 1} \nu(r)$ $=-\infty$. Let $u$ be a continuous subharmonic function on $\Delta$. If $S$ is a second category subset of $C$ such that

$$
\limsup _{\substack{|z| \rightarrow 1 \\ z \in \mathscr{S}_{\eta} \cap \Delta}} u(z)-\nu(|z|)<+\infty, \quad \eta \in S,
$$

then $u \equiv-\infty$.

For the proof, note that if a subharmonic function $u$ on $\Delta$ is continuously $-\infty$ at each point of a nonempty open $\operatorname{arc} A$ of $C$, then $u \equiv-\infty$ as is seen using simple harmonic measure estimates. When $f$ is analytic, Theorem 2 is easily subsumed under Theorem 4. In fact, letting $\nu=\log \mu, u=\log |f|$, and observing that $f(z)=$ $O[\mu(|z|)], z \in \mathbb{S}_{\eta}$, for each $\eta \in S$ implies (1), we see that the conclusion $u \equiv-\infty$ guarantees that $f \equiv 0$. Finally, we remark that in the case when $\&$ is the image of a Jordan arc $\gamma$ such that $|\gamma|$ is strictly increasing, Theorems 2 and 4 are seen to be sharp when $\mu$ and $\nu$ are monotonic using only a slight modification of the construction used by P. Gauthier (see [5, Theorem B]) to show that Theorem 1 is sharp.

\section{REFERENCES}

1. K. F. Barth and W. J. Schneider, An asymptotic analog of the $F$. and M. Riesz radial uniqueness theorem, Proc. Amer. Math. Soc. 22 (1969), 53-54.

2. T. L. Hayden and T. J. Suffridge, Biholomorphic maps in Hilbert space have a fixed point, Pacific J. Math. 38 (1971), 419-422.

3. P. J. Rippon, The boundary cluster sets of subharmonic functions, J. London Math. Soc. (2) 17 (1978), 469-479.

4. A A radial uniqueness theorem for meromorphic functions, unpublished.

5. K. F. Tse, An analog of the Lusin-Privaloff radial uniqueness theorem, Proc. Amer. Math. Soc. 25 (1970), 310-312.

Department of Mathematics, University of Maryland, College Park, Maryland 20742

Current address: Department of Mathematics, Wayne State University, Detroit, Michigan 48202 